\section{Fatores associados ao uso de bicicleta como meio de transporte em uma cidade de médio porte}

\section{Factors associated with the bicycle use for transportation in a medium-sized city}

\author{
Inaian Pignatti Teixeira ${ }^{1}$ \\ Priscila Missaki Nakamura ${ }^{1}$ \\ Bruno Paula Caraça Smirmaul ${ }^{1}$ \\ Rômulo Araújo Fernandes ${ }^{2}$ \\ Eduardo Kokubun
}

\section{Resumo}

O objetivo do presente estudo foi analisar a prevalência do uso da bicicleta como meio de transporte (MT) entre homens e mulheres e sua associação com fatores pessoais, educacionais e financeiros. Trata-se de um estudo transversal de base populacional, com amostra composta por 1588 adultos residentes no município de Rio Claro-SP. A prevalência de uso da bicicleta como MT foi de $28,3 \%$, sendo que entre os homens a prevalência $(38,3 \%)$ foi maior do que entre as mulheres $(21,0 \%)$. Os resultados indicam que idosos e pessoas com alta renda são menos propensas a fazerem uso desse MT. Além disso, observou-se que alguns fatores pessoais associam-se com o uso de bicicleta de forma distinta entre os sexos. Entre as mulheres, aquelas classificadas como obesas e não trabalhadoras apresentaram menores valores de odds para uso de bicicleta. Já entre os homens, a maior escolaridade foi associada de forma inversa com o uso de bicicleta. Tais resultados podem nortear intervenções de promoção do uso de bicicleta como MT que foquem principalmente nesses grupos com menores índices de utilização.

\section{Palavras-chave}

Atividade Física; Transportes; Adulto

\begin{abstract}
The purpose of the present study was to analyze the prevalence of bicycle use for transportation between men and women and its association with personal, educational and financial factors. It is a population-based study with sample of 1588 adults living in Rio Claro city - SP - Brazil. The prevalence of bicycle use for transportation was $28.3 \%$, in which the prevalence was higher among men (38.3\%) than among women (21.0\%). The results indicate that elderly and people with high income are less likely to use this kind of transport. Furthermore, it was observed that some personal factors are differently associated with bicycle use between genders. Among women, those classified as overweight and non-workers presented lower odds values for bicycle use. Among men, higher education level was inversely associated with bicycle use. Such results can guide promotion interventions for bicycle use for transportation focusing mainly in these groups with lower indexes for use.
\end{abstract}

\section{Keywords}

Motor Activity; Transportation; Adult.
Rev Bras Ativ Fis Saúde p. 698-710

DOl:

http://dx.doi.org/10.12820/rbafs.v.18n6p698

1 Universidade Estadual Paulista-Campus de Rio Claro-SP

2 Universidade Estadual Paulista- Campus de Presidente Prudente 


\section{INTRODUÇÃO}

O uso crescente dos automóveis como principal meio de locomoção vêm causando sérios problemas no sistema de transporte urbano, como congestionamentos ${ }^{1} \mathrm{e}$ poluição do ar e sonora ${ }^{2}$. Assim, o transporte ativo, principalmente na forma de caminhada e ciclismo, tem sido considerado como uma ótima alternativa para minimizar tais problemas ${ }^{3}$.

Além dos benefícios ambientais, o transporte por meio de bicicleta ou caminhada contribuem em diversos aspectos da saúde, como prevenção e tratamento de doenças cardiovasculares, alguns casos de câncer, redução da mortalidade por causas gerais e controle de peso ${ }^{4,5}$. Considerando que a atividade física (AF) no domínio doméstico e no trabalho são pouco suscetíveis às intervenções de políticas públicas $^{6}$, e que a $\mathrm{AF}$ realizada no período de tempo livre encontra-se estabilizada há mais de 20 anos $^{7}$, estratégias que promovam um aumento do tempo de $\mathrm{AF}$ no domínio do transporte vêm recebendo crescente atenção mundial ${ }^{8,9}$. Adicionalmente, vale destacar que um aumento da AF no domínio do transporte contribui para aumentar o tempo total de AF, colaborando para que indivíduos atinjam as recomendações de AF para promoção e manutenção da saúde ${ }^{10}$.

Dentre todas as modalidades de transporte ativo, a bicicleta é especialmente atrativa, por ser espacialmente econômica, de fácil manuseio, barata manutenção, com possibilidade de integração com outros meios de transportes, além de ser acessível a todas as idades e classes sociais ${ }^{11}$. Além disso, a substituição do automóvel pela bicicleta é bastante atrativa, principalmente para as pessoas que necessitam percorrer distâncias curtas e médias, de até $3,2 \mathrm{~km}^{12}$.

Dados nacionais apontam que $17 \%$ das pessoas usam a bicicleta como meio de transporte para atividades rotineiras, e apenas $8 \%$ a considera como o principal meio de deslocamento na cidade ${ }^{13}$, enquanto até $25 \%$ de todos os deslocamentos são feitos usando-se a bicicleta em outros países, principalmente europeus ${ }^{14}$. Dessa forma, seria interessante a criação de estratégias não apenas para aumentar o número de usuários da bicicleta como meio de transporte no cenário nacional, mas também para intensificar esse comportamento em pessoas que já a utilizam esporadicamente.

Vale destacar que homens tendem a utilizar mais a bicicleta como meio de transporte do que mulheres, tanto no Brasi $1^{15}$ como em outras localidades do mun$\mathrm{do}^{16,17}$. Além disso, Garrard e colaboradores ${ }^{18}$ destacam que os fatores associados ao uso de bicicleta como meio de transporte são distintos entre os sexos, reforçando a necessidade de se analisar tais fatores separadamente entre homens e mulheres.

Assim, identificar o perfil dos usuários da bicicleta como meio de transporte, bem como seus fatores associados, pode tornar futuras intervenções mais assertivas e eficazes. Portanto, o objetivo do presente estudo foi analisar a prevalência do uso da bicicleta como meio de transporte em uma cidade nacional de porte médio, bem como traçar o perfil desses usuários. Além disso, objetivou-se analisar os fatores pessoais, educacionais e financeiros associados ao uso de bicicleta de maneira estratificada por sexo. Dentro do nosso conhecimento, esse é o primeiro estudo nacional a investigar os fatores associados ao uso de bicicleta como meio de transporte para homens e mulheres de forma isolada.

\section{METODOLOGIA}

\section{Local do Estudo}

Estudo transversal de base populacional realizado de Outubro de 2007 a Novem- 
bro de 2008, com amostra composta por adultos, com mais de 20 anos, de ambos os sexos e residentes na zona urbana do município de Rio Claro - SP. O município de Rio Claro está localizado na região sudeste do estado de São Paulo, sendo sua área territorial de $1498 \mathrm{~km}^{2}$, área urbana de $499 \mathrm{~km}^{2}{ }^{19}$ e com relevo considerado plano com uma variação de aproximadamente 225 metros entre altura mínima (500m) e máxima (725m). Rio Claro apresenta 187.637 habitantes, sendo a maioria mulheres (51,3\%). A renda per capta da cidade gira em torno de 21 mil reais/ ano $^{19}$ e o Índice de Desenvolvimento Humano (IDH) em 2011 era de 0,825.

Os critérios de exclusão para o presente estudo foram: indivíduos institucionalizados (hospitais, instituições de longa permanência - asilos, prisões) e indivíduos com incapacidade mental. O estudo foi aprovado pelo Comitê de Ética em Pesquisa envolvendo seres humanos, do Instituto de Biociências da Universidade Estadual Paulista - UNESP, Campus de Rio Claro (processo: 8592). Os sujeitos foram informados sobre os objetivos do estudo e assinaram o Termo de Consentimento Livre e Esclarecido.

\section{Amostragem}

Para o cálculo do tamanho amostral foi considerado um erro amostral de $5 \%$ e prevalência de $50 \%$ de usuários de bicicleta como meio de transporte na população adulta de Rio Claro - SP. A amostra foi estimada em 764 participantes, calculada através da fórmula: $n=\left[N \cdot Z^{2} \cdot p \cdot(1-p)\right] \cdot\left[d^{2} \cdot(N-1)+Z^{2} \cdot p \cdot(1-p)\right]^{-1}$, sendo: $N=$ população de Rio Claro - SP, com 20 anos ou mais de idade, aproximadamente 128.000 pessoas; $Z$ = nível de significância (estabelecido em 95\%); $p$ = prevalência esperada do fenômeno a ser investigado; $d$ = erro amostral tolerável. Como a amostra foi realizada por conglomerados (setores censitários), o número de participantes foi duplicado, alcançando-se 1528 adultos.

O processo de amostragem foi realizado em três estágios: a) seleção dos setores censitários; b) seleção dos domicílios; c) seleção dos moradores com mais de 20 anos de idade. De acordo com o censo do IBGE de 2000 a cidade de Rio Claro apresentava 200 setores censitários catalogados. Por meio de sorteio foram selecionados os setores urbanos de número ímpar, que resultou em 99 setores. Destes, dois não são elegíveis (setor 27 e 45), segundo o Instituto Brasileiro de Geografia e Estatística (IBGE), resultando em 97 setores. Para completar o número de 100 setores (metade do total) foram acrescentados, atendendo o critério de distribuição (que abrangessem regiões equidistantes entre si e, portanto com características distintas), os setores de número 50, 100 e 150. Em seguida, 7 domicílios de cada setor foram selecionados $(\mathrm{n}=700)$ (estágio b) e todos os moradores com 20 anos ou mais foram incluídos na amostra, respeitando-se os critérios de exclusão (estágio c).

\section{Variável dependente: Ciclismo}

Para acessar informações relativas ao uso de bicicleta como meio de transporte, foram utilizadas duas questões do Questionário Internacional de Atividade Física (IPAQ) em sua versão longa. As perguntas utilizadas foram: "Quantos dias por semana o(a) Sr(a) usa a bicicleta para ir de um lugar a outro como forma de deslocamento?" e, caso a pessoa respondesse um ou mais dias era feito a pergunta "Quanto tempo no total o(a) Sr(a) pedala por dia por mais de 10 min?”. Para análise dos dados os sujeitos foram divididos em dois grupos, sendo um composto pelos usuários (ciclistas; $\geq 10$ minutos por semana) e o outro formado pelos não usuários (não-ciclistas; $\leq 9$ minutos por semana) de bicicleta como meio de transporte. 


\section{Variáveis independentes}

O perfil dos ciclistas e da amostra foi estruturado a partir de questões auto reportadas sobre idade (20 a 39 anos, 40 a 59 anos e acima de 60 anos de idade), sexo, peso e estatura para o cálculo do Índice de Massa Corporal (IMC) [normal ( $<25$ $\mathrm{kg} / \mathrm{m}^{2}$ ), sobrepeso $\left(25\right.$ a $\left.29,99 \mathrm{~kg} / \mathrm{m}^{2}\right)$ e obeso $\left(\geq 30 \mathrm{~kg} / \mathrm{m}^{2}\right)$ ], estado civil (solteiro, casado/amasiado e viúvo/divorciado), escolaridade (até a $4^{\mathrm{a}}$ série, segundo grau incompleto e segundo grau completo ou superior), situação trabalhista (não trabalha e trabalha) e classe econômica (D-E, C, A-B). Para acessar a classe econômica foi utilizado o questionário elaborado pela Associação Brasileira de Empresas de Pesquisa, que se baseia em uma estimativa do poder de compra das pessoas e famílias urbanas.

Antes de iniciar a coleta de dados os instrumentos foram testados em um setor censitário que não fazia parte da amostra, com objetivo de padronizar os procedimentos da coleta. Em seguida, foram selecionados dez indivíduos, de ambos os sexos, com segundo grau completo para serem os entrevistadores. Eles receberam um treinamento de 40 horas que englobava estudo teórico das técnicas de entrevista, dramatizações e aplicação do questionário utilizando um manual de instruções elaborado especificamente para tal fim. Além dos entrevistadores, também foram contratados dois digitadores de ambos os sexos que tinham o segundo grau completo e conhecimento básico em base de dados no programa EPI INFO.

O questionário foi aplicado em forma de entrevista individual e o tempo de duração média para aplicação foi de 60 minutos. $\mathrm{O}$ controle de qualidade, que consistiu em confirmar a veracidade das informações coletadas pelos entrevistadores, foi realizado pela re-visita de $10 \%$ dos domicílios pelos pesquisadores. Foi estabelecido como recusa quando o morador se recusasse a responder o questionário ou após cinco visitas (três do entrevistador e duas do pesquisador).

\section{Análises estatísticas}

Foi realizada uma análise descritiva estratificada por sexo, sendo os resultados expressos em valores percentuais com seus respectivos intervalos de confiança de 95\% (IC95\%). Para as medidas de associação entre o uso de bicicleta e as variáveis independentes (idade, IMC, estado civil, escolaridade, situação trabalhista e classe econômica) foi realizada uma regressão logística múltipla (expressa como valores de Odds Ratio (OR) e seus intervalos de confiança de 95\%), estratificada por sexo. O modelo de regressão múltipla foi composto por todas as variáveis independentes, sendo inseridas no modelo de acordo com o valor de p nas análises de qui-quadrado (do menor para o maior). Caso a variável independente piorasse o ajuste do modelo, baseado no $\mathrm{R}^{2}$ de Cox \& Snell, a mesma seria descartada do modelo final. O modelo final foi composto e ajustado por todas as variáveis independentes, uma vez que todas atenderam as exigências das etapas anteriores. Para todas as análises foi utilizado o software SPSS versão 17.0.

\section{RESULTADOS}

No presente estudo foram abordados 1464 domicílios, dos quais 960 (66\%) foram elegíveis para o estudo, sendo que os demais apresentaram problemas na fase do arrolamento, como endereço errado/incompleto, casas desocupadas, comércios, etc. Dentre os 960 domicílios elegíveis, 800 (83\%) tiveram seus moradores entrevistados com sucesso e as recusas (indivíduos que não responderam ao questionário; relato de falta de tempo; não foram encontrados nas cinco tentativas de 
entrevistas; alegaram problemas de saúde para não responder) somaram um total de $17 \%$. Assim, foram entrevistados 1588 sujeitos, sendo 917 mulheres $(57,7 \%)$ e 671 homens (42,3\%), resultando em uma média de 1,9 entrevistas por domicílio.

A Tabela 1 apresenta o perfil da amostra total estratificada por sexo, segundo idade, estado civil, classe econômica, escolaridade, IMC e situação trabalhista. Nota-se que houve uma proporção diferente entre os homens e mulheres para as variáveis estado civil, classe econômica, escolaridade e situação trabalhista. Vale destacar que existe uma menor proporção de homens nas classes econômicas D e E quando comparado com as mulheres, assim como para escolaridade menor que a $4^{\mathrm{a}}$ série. Em contrapartida, a proporção de trabalhadores homens é maior do que as trabalhadoras (Tabela 1 ).

Tabela 1 - Fatores pessoais, educacionais/financeiros da amostra total estratificada por sexo ( $n=1588$, Rio Claro - SP, 2007-2008).

\begin{tabular}{|c|c|c|c|c|c|c|c|c|c|}
\hline & \multicolumn{3}{|c|}{ Mulheres } & \multicolumn{3}{|c|}{ Homens } & \multicolumn{3}{|c|}{ Amostra total } \\
\hline & \multirow[t]{2}{*}{$\%$} & \multicolumn{2}{|c|}{ (IC95\%) } & \multirow[t]{2}{*}{$\%$} & \multicolumn{2}{|c|}{ (IC95\%) } & \multirow[t]{2}{*}{$\%$} & \multicolumn{2}{|c|}{ (IC95\%) } \\
\hline \multicolumn{7}{|l|}{ Faixa etária } & & & \\
\hline 20 a 39 anos & 39,3 & $(36,5$ & $42,1)$ & 41,9 & $(39,0$ & $44,7)$ & 40,4 & $(38,0$ & $42,8)$ \\
\hline 40 a 59 anos & 36,6 & $(33,8$ & $39,4)$ & 38,2 & $(35,3$ & $41,0)$ & 37,2 & $(34,8$ & $39,6)$ \\
\hline 60 anos ou mais & 24,1 & $(21,6$ & $26,6)$ & 20,0 & $(17,6$ & $22,3)$ & 22,4 & $(20,3$ & $24,5)$ \\
\hline \multicolumn{10}{|l|}{ Estado Civil } \\
\hline Solteiro & 16,9 & $(14,7$ & $19,1)$ & 23,8 & $(21,4$ & $26,3)$ & 19,8 & $(17,8$ & $21,8)$ \\
\hline Casado/amasiado & 61,7 & $(58,9$ & $64,5)$ & 68,6 & $(65,9$ & $71,3)$ & 65,2 & $(62,9$ & $67,5)$ \\
\hline Viúvo/divorciado & 21,3 & $(18,9$ & $23,7)$ & 7,6 & $(6,1$ & $9,1)$ & 15,0 & $(13,2$ & $16,8)$ \\
\hline \multicolumn{10}{|l|}{ Índice de Massa Corporal } \\
\hline Normal & 49,3 & $(46,4$ & $52,2)$ & 46,9 & $(44,0$ & $49,8)$ & 48,3 & $(45,8$ & $50,8)$ \\
\hline Sobrepeso & 33,0 & $(30,3$ & $35,7)$ & 36,5 & $(33,7$ & $39,3)$ & 34,5 & $(32,2$ & $36,8)$ \\
\hline Obeso & 17,7 & $(15,5$ & $19,9)$ & 16,6 & $(14,4$ & $18,8)$ & 17,2 & $(15,3$ & $19,1)$ \\
\hline Classe Econômica & & & 0 & & & & & & \\
\hline$D, E$ & 20,5 & $(18,1$ & $22,8)$ & 14,9 & $(12,8$ & $17,0)$ & 18,1 & $(16,2$ & $20,0)$ \\
\hline C & 46,6 & $(43,7$ & $49,5)$ & 47,8 & $(44,9$ & $50,7)$ & 47,1 & $(44,6$ & $49,6)$ \\
\hline$A, B$ & 32,9 & $(30,3$ & $35,7)$ & 37,3 & $(34,5$ & $40,1)$ & 34,8 & $(32,5$ & $37,1)$ \\
\hline \multicolumn{10}{|l|}{ Escolaridade } \\
\hline Até a $4^{\mathrm{a}}$ série & 40,7 & $(37,9$ & $43,6)$ & 31,4 & $(28,7$ & $34,1)$ & 36,8 & $(34,4$ & $39,2)$ \\
\hline $\begin{array}{l}2^{\circ} \text { grau } \\
\text { incompleto }\end{array}$ & 19,5 & $(17,2$ & $21,8)$ & 17,4 & $(15,2$ & $19,6)$ & 18,7 & $(16,8$ & $20,6)$ \\
\hline $\begin{array}{l}2^{\circ} \text { grau completo/ } \\
\text { ensino superior }\end{array}$ & 39,7 & $(36,9$ & $42,6)$ & 51,1 & $(48,2$ & $54,0)$ & 44,5 & $(42,1$ & $46,9)$ \\
\hline \multicolumn{10}{|l|}{ Situação Trabalhista } \\
\hline Não trabalha & 53,1 & $(50,2$ & $56,0)$ & 26,6 & $(24,0$ & $29,1)$ & 41,9 & $(39,5$ & $44,3)$ \\
\hline Trabalha & 46,9 & $(44,0$ & $49,8)$ & 73,4 & $(70,9$ & $76,0)$ & 58,1 & $(55,7$ & $60,5)$ \\
\hline
\end{tabular}

Dos 1588 entrevistados, 449 (28,3\%) utilizam a bicicleta como meio de transporte por pelo menos 10 minutos por semana, sendo 257 homens e 192 mulheres. Foi observada uma maior proporção de homens $(38,3 \%$, IC95\% = 35,3-41,3\%) que usam desse meio de transporte quando comparado com as mulheres $(21,0 \%, \mathrm{IC} 95 \%=18,4-23,5 \%)$. 
A Figura 1 apresenta o perfil dos ciclistas estratificado por sexo segundo fatores pessoais e educacionais/financeiros. De uma forma geral, nota-se que o perfil dos ciclistas homens e ciclistas mulheres é similar, sendo composto principalmente por indivíduos com idade entre 20 e 39 anos, casados ou amasiados, com IMC normal, de classe econômica "C", com escolaridade igual ou superior ao $2^{\circ}$ grau completo e que trabalham.
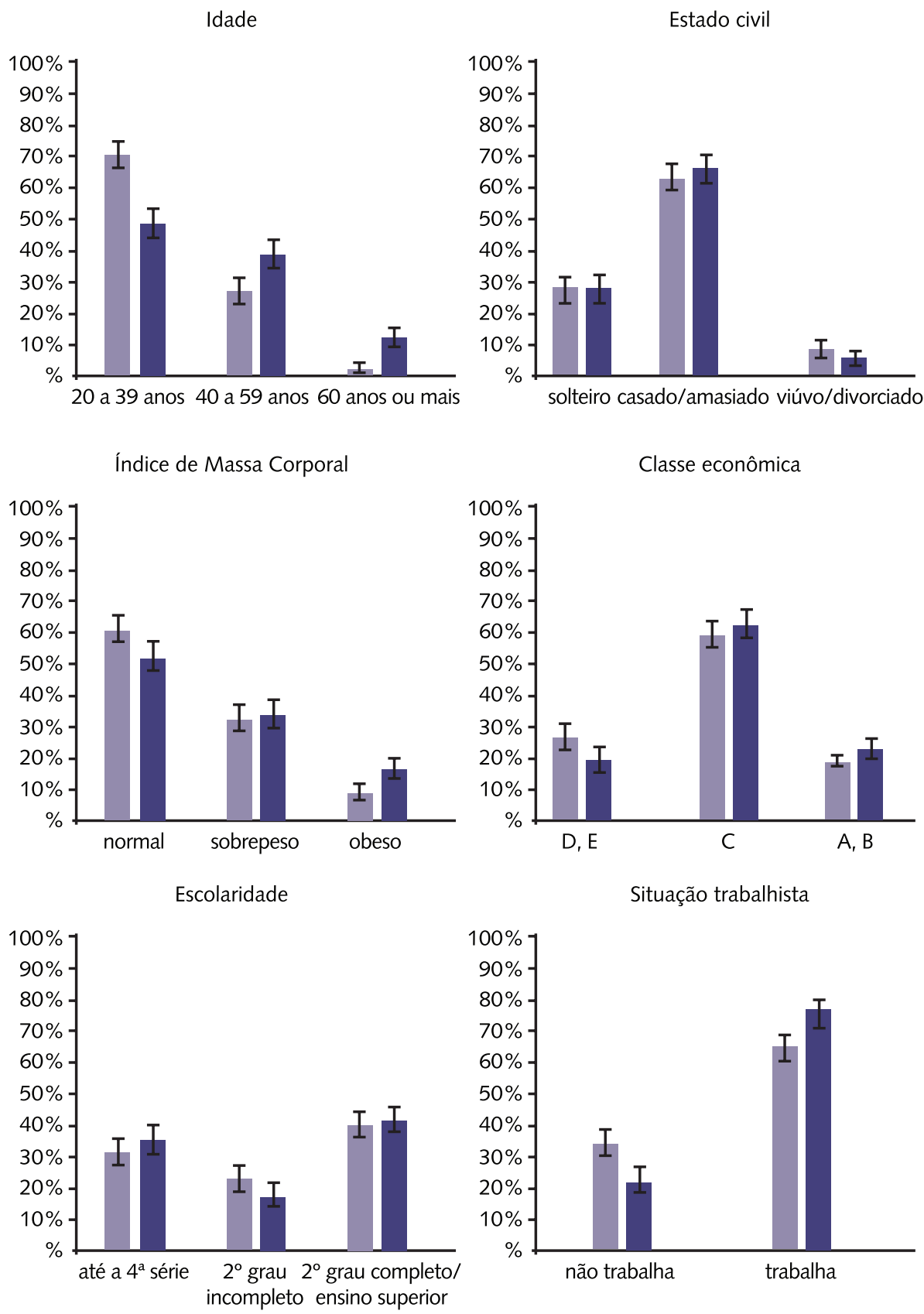

Ciclistas (mulheres) Ciclistas (homens)

Figura 1 - Perfil dos ciclistas homens e mulheres segundo fatores pessoais e fatores educacionais/financeiro. Nota: as barras indicam a prevalência e as linhas de erro indicam os intervalos de confiança de $95 \%$

A Tabela 2 apresenta os resultados da análise de qui-quadrado entre o uso de bicicleta como meio de transporte e os fatores pessoais, educacionais/financeiros, estratificada por sexo. Nota-se que entre as mulheres, todas as variáveis indepen- 
dentes apresentaram associações significativas $(p<0,05)$ com o uso de bicicleta como meio de transporte. Entre os homens foi verificado um comportamento similar, exceto pelo Índice de Massa Corporal que apresentou um valor de p= 0,253.

Tabela 2 - Qui-quadrado univariado entre o uso de bicicleta como meio de transporte e os fatores pessoais, educacionais/financeiros, estratificado por sexo ( $n=1588$, Rio Claro-SP, 2007-2008).

\begin{tabular}{lcccccc} 
& \multicolumn{3}{c}{ Mulheres } & \multicolumn{3}{c}{ Homens } \\
& $\mathrm{x}^{2}$ & $\mathrm{gl}$ & $\mathrm{p}$ & $\mathrm{x}^{2}$ & $\mathrm{gl}$ & $\mathrm{p}$ \\
\hline Faixa etária & 112,06 & 2 & $<0,01$ & 16,40 & 2 & $<0,01$ \\
\hline Estado Civil & 21,77 & 2 & $<0,01$ & 10,89 & 2 & $<0,01$ \\
\hline Índice de Massa Corporal & 14,38 & 2 & $<0,01$ & 2,75 & 2 & 0,25 \\
\hline Classe Econômica & 23,870 & 2 & $<0,01$ & 41,853 & 2 & $<0,01$ \\
\hline Escolaridade & 6,156 & 2 & 0,04 & 9,156 & 2 & 0,01 \\
\hline Situação Trabalhista & 34,22 & 1 & $<0,01$ & 3,93 & 1 & 0,03 \\
\hline
\end{tabular}

A Tabela 3 apresenta os resultados da análise de regressão logística da associação entre o uso de bicicleta como meio de transporte e os fatores pessoais e educacionais/financeiros, estratificados por sexo. Para as mulheres, os resultados indicam uma associação entre faixa etária, classe econômica, IMC e situação trabalhista com o uso de bicicleta como meio de transporte. Nota-se que as mulheres com mais de 40 anos de idade ( 40 a 59 anos e 60 anos ou mais), pertencentes às classes econômicas $\mathrm{A}$ e $\mathrm{B}$, obesas e que não trabalham apresentaram menores valores de odds para o uso de bicicleta quando comparadas com as mulheres com menos de 20 anos, das classes econômicas D e E, com IMC normal e que trabalham (Tabela 3).

Para os homens, os fatores associados ao uso de bicicleta como meio de transporte foram faixa etária, classe econômica e escolaridade. Os resultados da regressão logística apontam que os homens com mais de 60 anos, com $2^{\circ}$ grau completo/ superior e pertencentes às classes econômicas $\mathrm{A}$ e $\mathrm{B}$ exibiram menores valores de odds para o uso de bicicleta quando comparados com os homens mais jovens ( 20 a 40 anos), com escolaridade menor ou igual à $4^{\mathrm{a}}$ série e pertencentes às classes econômicas D e E (Tabela 3).

Tabela 3 - Análise de Regressão Logística múltipla, sendo a variável dependente o uso de pelo menos 10 minutos por semana de bicicleta no domínio deslocamento, estratificada por sexo $(n=1588$, Rio Claro-SP, 2007-2008).

\begin{tabular}{lcccccc}
\hline & \multicolumn{5}{c}{ Mulheres } & \multicolumn{3}{c}{ Homens } \\
& OR & $\left(\mathrm{IC}_{95 \%}\right)$ & $\mathrm{P}$ & $\mathrm{OR}$ & $\left(\mathrm{IC}_{95 \%}\right)$ & $\mathrm{P}$ \\
\hline Faixa etária & & & $<0,001$ & & & 0,001 \\
\hline 20 a 39 anos & 1 & & & 1 & & \\
\hline 40 a 59 anos & 0,34 & $(0,22-0,53)$ & & 0,94 & $(0,61-1,46)$ & \\
\hline 60 anos ou mais & 0,03 & $(0,01-0,09)$ & & 0,34 & $(0,18-0,65)$ & \\
\hline Estado civil & & & 0,129 & & & 0,280 \\
\hline Solteiro & 1 & & & 1 & & \\
\hline Casado/amasiado & 0,70 & $(0,44-1,12)$ & & 0,76 & $(0,47-1,23)$ & \\
\hline Viúvo/divorciado & 1,12 & $(0,58-2,16)$ & & 1,15 & $(0,59-2,22)$ & \\
\hline IMC & & & 0,042 & & & 0,613 \\
\hline Normal & 1 & & & 1 & & \\
\hline Sobrepeso & 1,01 & $(0,66-1,54)$ & & 0,82 & $(0,56-1,20)$ & \\
\hline Obeso & 0,47 & $(0,26-0,87)$ & & 0,88 & $(0,54-1,43)$ & \\
\hline
\end{tabular}




\begin{tabular}{lcccccc}
\hline & \multicolumn{3}{c}{ Mulheres } & \multicolumn{3}{c}{ Homens } \\
\hline & OR & $\left(\mathrm{IC}_{95 \%}\right)$ & $\mathrm{p}$ & $\mathrm{OR}$ & $\left(\mathrm{IC}_{95 \%}\right)$ & $\mathrm{P}$ \\
\hline Classe econômica & & & $<0,001$ & & & $<0,001$ \\
\hline D e E & 1 & & & 1 & & \\
\hline C & 0,78 & $(0,49-1,27)$ & & 1,23 & $(0,75-2,01)$ & \\
\hline A e B & 0,32 & $(0,17-0,58)$ & & 0,47 & $(0,26-0,83)$ & \\
\hline Escolaridade & & & 0,279 & & & 0,042 \\
\hline Até a 4 4 série & 1 & & & 1 & & \\
\hline $2^{\circ}$ grau incompleto & 0,74 & $(0,43-1,24)$ & & 0,63 & $(0,37-1,06)$ & \\
\hline $2^{\circ}$ grau completo/superior & 0,66 & $(0,39-1,10)$ & & 0,54 & $(0,33-0,88)$ & \\
\hline Situação Trabalhista & & & 0,006 & & & 0,560 \\
\hline Trabalha & 1 & & & 1 & & \\
\hline Não trabalha & 0,57 & $(0,38-0,85)$ & & 0,87 & $(0,55-1,37)$ & \\
\hline
\end{tabular}

\section{DISCUSSÃO}

O objetivo do presente estudo foi traçar o perfil dos usuários de bicicleta como meio de transporte em uma cidade de porte médio, e identificar os fatores pessoais e educacionais/financeiros associados com essa modalidade de transporte, de maneira estratificada por sexo. Os principais resultados mostram que o perfil dos ciclistas homens e mulheres são similares, sendo composto principalmente por indivíduos trabalhadores, com idade entre 20 e 39 anos, casados ou amasiados, com IMC normal, de classe econômica "C", com escolaridade igual ou superior ao $2^{\circ}$ grau completo. Já os fatores associados ao uso da bicicleta como meio de transporte são distintos entre os sexos sendo que, para homens, faixa etária, classe econômica e escolaridade associaram-se significativamente, e para as mulheres, faixa etária, classe econômica, IMC e situação trabalhista.

A prevalência de uso de bicicleta como meio de transporte no presente estudo foi de $28,3 \%$. Este valor está bem acima dos normalmente encontrados em outras cidades nacionais, como Vitória (8,8\%), Curitiba $(9,6 \%)$ e Recife $(16,0 \%)^{15}$. Essa proporção encontrada em Rio Claro equipara-se inclusive a países europeus como a Dinamarca (19\%) e Países baixos $(25 \%)^{20}$, que dedicam grandes esforços para a promoção deste meio de transporte. Especula-se que essa alta prevalência de uso de bicicleta na cidade de Rio Claro seja em virtude da relativamente pequena área urbana $\left(498 \mathrm{~km}^{2}\right)$, com relevo plano (variação de aproximadamente 225 metros), aliado à existência de facilitadores para o tráfego de bicicletas (ciclovias, ciclofaixas e bicicletários) e pela ineficiência do sistema de transporte da cidade.

Homens apresentaram maior prevalência de uso de bicicleta como meio de transporte quando comparado com as mulheres (38,3\%; IC95\%=35,3-41,3 x 21,0\%; IC95\%=18,4-23,5, respectivamente). Diversos estudos também apontam essa tendência de maior utilização de bicicleta entre os homens ${ }^{15-17}$. Em contrapartida, Garrard e colaboradores ${ }^{21}$, afirmam que a relação entre o sexo e o uso de bicicleta se dá de diferentes formas entre os países, sendo que, em países com altas taxas de ciclistas, esta atividade é tão comum entre as mulheres quanto nos homens. Porém, no presente estudo, apesar da alta proporção de ciclistas em geral $(28,3 \%)$, não foi observada essa equiparidade entre os sexos.

$\mathrm{Na}$ literatura, a relação entre a idade e o uso de bicicleta não é clara, sendo que alguns estudos apontam um declínio dos níveis de ciclismo com o passar da ida$\mathrm{de}^{15,16,22,23} \mathrm{e}$ outros não ${ }^{17,24,25}$. No presente estudo, observou-se que, para ambos os sexos, os idosos (mais de 60 anos) apresentam menores valores de odds para o uso 
de bicicleta quando comparados aos mais novos (20 a 40 anos), sendo que nas mulheres idosas esse valor é $97 \%$ menor e para os homens idosos $66 \%$ menor. Adicionalmente, as mulheres com idade entre 40 e 59 anos também apresentam menores valores de odds $(\mathrm{RO}=0,34 ; \mathrm{IC} 95 \%=0,22-0,53)$ para o uso de bicicleta quando comparado às mais novas. Segundo Lohmann $\&$ Rolle $^{26}$, os idosos são mais suscetíveis às limitações físicas, comprometendo o uso de bicicleta e, em função disto, a idade frequentemente é relatada como uma barreira para o não uso. Já Sener, Eluru \& Bhat ${ }^{16}$ atribuem essa diferença ao fato que os mais jovens possuiriam uma maior consciência sobre os efeitos benéficos do uso de bicicleta frente ao meio ambiente e, por consequência, usariam a bicicleta para locomoções adicionais, além da finalidade de lazer/exercício. Outro fator contribuidor pode ser a melhor percepção frente aos facilitadores para o uso da bicicleta (ex: ciclovia, ciclofaixa, sinalização) pelos homens e as pessoas mais jovens em relação às mulheres e pessoas idosas ${ }^{16}$.

Não foi observada uma associação significativa entre o estado civil e o uso de bicicleta como meio de transporte, tanto para mulheres como para homens. Adicionalmente, outros estudos realizados em 3 cidades brasileiras ${ }^{15}$ e 3 cidades dos Estados Unidos ${ }^{17}$ não observaram essa associação. Aparentemente, o estado civil agrega outras variáveis que podem ser determinantes do uso de bicicleta e que merecem o interesse de futuros estudos.

Com relação ao IMC, não foi observada uma associação significativa entre os homens, porém, entre as mulheres, observou-se que, aquelas classificadas como obesas, apresentam menores valores de odds $(\mathrm{RO}=0,47 \mathrm{IC} 95 \%=0,26-0,87)$ para o uso de bicicleta em comparação com as mulheres de IMC normal. Outros trabalhos, como o de Sallis e colaboradores ${ }^{17}$, também verificaram uma associação inversamente proporcional entre o IMC e a frequência de uso de bicicleta. Comportamento similar também foi observado em outros estudos no Brasil ${ }^{15}$ e em 17 países da América do Norte, Europa e Austrália ${ }^{14}$. Vale destacar que a natureza transversal desses estudos impossibilita estabelecer a relação causa e efeito, porém, acredita-se que o uso prolongado da bicicleta como meio de transporte pode acarretar benefícios no controle do peso corporal.

A relação entre a situação trabalhista e o uso de bicicleta não é clara na literatura. Owen e colaboradore ${ }^{27}$ encontraram que, para a população Belga, as pessoas que trabalham apresentam menores valores de odds $(\mathrm{RO}=0,54 ; \mathrm{IC} 95 \%=0,33-0,88)$ para o uso de bicicleta como meio de transporte quando comparado com as pessoas que não trabalham. Porém, esse comportamento não foi observado na população australiana $(\mathrm{RO}=1,25 ; \mathrm{IC} 95 \%=0,91-1,73)^{27}$. No presente estudo, entre os homens, também não foi observada tal associação, porém, entre as mulheres, o fato de não trabalhar resultou em menores valores de odds para o uso de bicicleta como meio de transporte $(\mathrm{RO}=0,57 \mathrm{IC} 95 \%=0,38-0,85)$. A inexistência de consenso sobre tal associação pode ser explicada devido às diferentes formas de análise (estratificada por sexo ou ajustada por sexo) e o não controle do tempo da jornada de trabalho e quantidade de esforço envolvido na atividade ocupacional, as quais que pode influenciar na opção pelo uso ou não da bicicleta como meio de transporte ${ }^{28}$.

No que se tange à escolaridade, estudos realizados no $\mathrm{Brasil}^{15} \mathrm{e}$ em outros países ${ }^{24,29}$ apontam uma relação inversa entre a escolaridade e uso de bicicleta como meio de transporte. Porém, no presente estudo, tal comportamento só foi observado entre os homens, sendo que os com maior escolaridade ( $2^{\circ}$ grau completo/ superior) apresentam menor valor de odds $(\mathrm{RO}=0,54 \mathrm{IC} 95 \%=0,33-0,88)$ para o uso da bicicleta como meio de transporte quando comparados com os homens 
que possuem escolaridade até a $4^{\mathrm{a}}$ série. Para as mulheres não houve associação significativa entre essa variável e o uso de bicicleta.

Os resultados referentes à relação entre o uso de bicicleta e a classe econômica apontam que, para ambos os sexos, as pessoas das classes A e B tiveram menores valores de odds quando comparados com as pessoas das classes $\mathrm{D}$ e E. Tais resultados corroboram os achados em outras cidades do Brasil ${ }^{15}$ e ao redor do mundo ${ }^{20,30}$. Em geral, ter maior renda reflete na maior possibilidade de gasto com transportes alternativos à bicicleta, inclusive com o investimento em um automóvel. A posse de carro, por sua vez, tem uma significativa associação inversa com o uso de bicicleta como meio de transporte, uma vez que as pessoas com posse de carro tendem a optar menos pelos deslocamentos ativos quando comparados com as pessoas sem a disponibilidade do mesmo ${ }^{29}$.

Particularmente no Brasil, ainda existe a cultura de que a bicicleta é um meio de transporte voltado para pessoas que não possuem condições de ter um automóvel. Em contrapartida, esse cenário se encontra em processo de mudança, frente à tendência mundial de conscientização dos efeitos benéficos do uso de bicicleta para o meio ambiente e a saúde ${ }^{8,9}$. Além disso, os países da América Latina e principalmente o Brasil, necessitam que a política de saúde e do transporte trabalhem juntas para aumentar a promoção do transporte ativo ${ }^{31}$.

Por fim, vale destacar que, além das características pessoais como idade, sexo, estado civil, escolaridade e renda, outros fatores como a cultura podem afetar o uso de bicicleta como meio de transporte ${ }^{32}$. Pucher e colaboradores ${ }^{32}$ destacam que os aspectos culturais específicos de países ou regiões podem interagir de maneira distinta frente ao uso de bicicleta como meio de transporte. Em países onde o uso da bicicleta é mais disseminado, as pessoas são mais propensas a utilizá-la, tem mais acesso aos equipamentos e aos conhecimentos necessários. Adicionalmente, os motoristas apresentam mais respeito para com o ciclista, em parte porque são mais propensos a também serem ciclistas ou pelo fato de conhecer outras pessoas que são ${ }^{32}$.

\section{LIMITAÇÕES}

O estudo apresentou algumas limitações, tais como, o sorteio dos setores censitários de forma conglomerada (ímpar ou par) ao invés do sorteio dos setores de forma separada. Entretanto, não houve diferença entre os setores ímpares e pares quando comparado o Índice de Paulista de Vulnerabilidade Social. Outro ponto a ser considerado é que o processo de amostragem resultou em uma maior proporção de mulheres em relação aos homens $(57,7 \%$ x 42,3\%) quando comparado aos dados do CENSO 2010 (51,3\% x 48,7\%), porém, como as análises foram realizadas estratificadas por sexo, eliminou-se uma possível supervalorização da amostra feminina. Por fim, sugerimos que futuros estudos avaliem também as barreiras e facilitadores para o uso da bicicleta, a fim de verificar quais suas interações com características pessoais.

\section{CONCLUSÃO}

O presente estudo verificou uma elevada prevalência de pessoas que usam a bicicleta como meio de transporte na cidade de Rio Claro - SP quando comparado a outras cidades nacionais e até cidades internacionais, consideradas casos bem sucedidos em relação a esse meio de transporte. $O$ perfil predominante do ciclista 
é composto por indivíduos trabalhadores, com idade entre 20 e 39 anos, casados ou amasiados, com IMC normal, de classe econômica "C", com escolaridade igual ou superior ao $2^{\circ}$ grau completo. Entretanto, essa associação é distinta entre os sexos, sendo que para os homens foram associados faixa etária, classe econômica e escolaridade e para as mulheres foram faixa etária, classe econômica, IMC e situação trabalhista.

Os diferentes fatores associados ao uso da bicicleta para homens e mulheres, demonstra a necessidade de preocupações distintas entre os sexos para a criação de intervenções, levando em consideração o nível de escolaridade, classe econômica, faixa etária, situação trabalhista e IMC. Intervenções que considerem esses fatores podem otimizar a sua efetividade em aumentar o uso da bicicleta entre os ciclistas. Além disso, é possível criar intervenções mais assertivas para a promoção do uso da bicicleta como meio de transporte para idosos, indivíduos com alta renda, homens com alta escolaridade, mulheres obesas e mulheres não trabalhadoras, uma vez que esses fatores foram associados de forma inversa com o uso de bicicleta.

Por fim, vale destacar que, estudos dessa natureza ainda são escassos no país, principalmente no que tange às diferenças entre homens e mulheres, e faz-se necessário construir um maior referencial teórico que sustente adequadamente o desenvolvimento de políticas públicas eficientes de estímulo do uso da bicicleta como meio de transporte no Brasil.

\section{Agradecimentos/Financiamentos}

A Fundação de Amparo à Pesquisa do Estado de São Paulo (FAPESP [Processo: 2012/18795-7]) pelo apoio financeiro para o desenvolvimento da pesquisa.

\section{REFERÊNCIAS}

1. Ogilvie D, Egan M, Hamilton V, Petticrew M. Promoting walking and cycling as an alternative to using cars: systematic review. BMJ [Internet]. 2004 Oct 2 [cited 2011 Aug 8];329(7469):763. Available from: http://www.pubmedcentral.nih.gov/articlerender. fcgi?artid=520994\&tool=pmcentrez\&rendertype=abstract

2. Nazelle A De, Nieuwenhuijsen MJ, Antó JM, Brauer M, Briggs D, Braun-fahrlander C, et al. Improving health through policies that promote active travel : A review of evidence to support integrated health impact assessment. Environ. Int. [Internet]. Elsevier Ltd; 2011;37(4):76677. Available from: http://dx.doi.org/10.1016/j.envint.2011.02.003

3. Ogilvie D, Bull F, Cooper A, Rutter H, Adams E, Brand C, et al. Evaluating the travel, physical activity and carbon impacts of a "natural experiment" in the provision of new walking and cycling infrastructure: methods for the core module of the iConnect study. BMJ Open [Internet]. 2012 Jan [cited 2012 Aug 4];2(1). Available from: http://www.pubmedcentral.nih. gov/articlerender.fcgi?artid=3274720\&tool=pmcentrez\&rendertype=abstract

4. Gordon-larsen P, Boone-heinonen J, Sidney S, Sternfeld B, Jacobs D, Lewis CE. Active Commuting and Cardiovascular Disease Risk. 2009;169(13):1216-23.

5. Rojas-rueda D, Nazelle A De, Tainio M. The health risks and benefits of cycling in urban environments compared with car use: health impact assessment study. BMJ. 2011;343(D4521):1-8.

6. Brasil. Ministério da Saúde. Avaliação de Efetividade de Programas de Atividade Física no Brasil. 1a Edição. Brasília; 2011.p. 65-6.

7. Hallal PC, Knuth AG, Reis RS, Rombaldi AJ, Iser BPM, Bernal RTI, et al. Tendências temporais de atividade física no Brasil. Rev. Bras. Epidemiol. 2011;14(1):53-60.

8. Pucher J, Dill J, Handy S. Infrastructure, programs, and policies to increase bicycling: an international review. Prev. Med. (Baltim). [Internet]. Elsevier Inc.; 2010 Jan [cited 2012 Jul 22];50 Suppl 1:S106-25. Available from: http://www.ncbi.nlm.nih.gov/pubmed/19765610 
9. Carnall D. Cycling and health promotion: a safer, slower urban road environment is the key. 2000. p. 2000.

10. Garber CE, Blissmer B, Deschenes MR, Franklin BA, Lamonte MJ. Quantity and Quality of Exercise for Developing and Maintaining Cardiorespiratory, Musculoskeletal, and Neuromotor Fitness in Apparently Healthy Adults: Guidance for Prescribing Exercise. Med. Sci. Sport. Exerc. 2011;43(7):1334-59.

11. Delabrida ZNC. A imagem e o uso da bicicleta: Um estudo entre moradores de Taguatinga [Internet]. UNIVERSIDADE DE BRASÍLIA; 2004. p. 67. Available from: https://doc-00-7g-docsviewer.googleusercontent.com/viewer/securedownload/ dsn1aovipa718461sfcf94nedj8q2p4u/2attarvk8gs8ul6pimhl7ecti5ii6vjq/1372085100000/ c210ZXM=/AGZ5hq8BgbJY1gwaOYx83cPOdNw6/WkdWbV1YVnNkR1J2Y1dGcGJueG1ZV0pwYjJSbFkzSnBjM1J2ZkdkNE9qUTRNR1E1W1daaE1UTXdZa$1 \mathrm{EwW} 1 \mathrm{RJ}$ ?docid=a5a68fdbe3818a56ca13d52205c191b9\%7Ce621624e 9c55e1ce220fbc37308df47b \&chan=EgAAAER4L0pgoEnNnaHtR\%2BdLO5hh/6xx4YRTvQpSWbL55rAi\&sec=AHSqidZkWIUjJHoZpbG2wEowgxXQI0zUf_vDIb1dAHLsfbGUAJmDtsagYw54ebrGSJuBFMNcjoFI\&a=gp\&filename

12. Pucher J, Dijkstra L. Promoting safe walking and cycling to improve public health: lessons from The Netherlands and Germany. Am. J. Public Health [Internet]. 2003 Sep;93(9):1509-16. Available from: http://www.pubmedcentral.nih.gov/articlerender. fcgi? artid=1448001\&tool=pmcentrez\&rendertype $=$ abstract

13. CNI-IBOPE. Retratos da Sociedade Brasileira: Locomoçao Urbana. 2011; Available from: http://www.ibope.com.br/pt-br/conhecimento/relatoriospesquisas/Lists/ RelatoriosPesquisaEleitoral/OPP 110004 - CNI Locomo\%C3\%A7\%C3\%A3o urbana.pdf

14. Bassett DR, Pucher J, Buehler R, Thompson DL, Crouter SE. Walking, cycling, and obesity rates in Europe, North America, and Australia. J. Phys. Act. Health [Internet]. 2008 Nov;5(6):795-814. Available from: http://www.ncbi.nlm.nih.gov/pubmed/19164816

15. Reis RS, Hino A a F, Parra DC, Hallal PC, Brownson RC. Bicycling and walking for transportation in three Brazilian cities. Am. J. Prev. Med. [Internet]. Elsevier Inc.; 2013 Feb [cited 2013 Sep 25];44(2):e9-17. Available from: http://www.ncbi.nlm.nih.gov/ pubmed/23332344

16. Sener IN, Eluru N, Bhat CR. An Analysis of Bicyclists and Bicycling Characteristics : Who, Why , and How Much are they Bicycling ? 88th Annu. Meet. Transp. Res. Board. Washington, DC.; 2009. p. 29.

17. Sallis JF, Conway TL, Dillon LI, Frank LD, Adams M a, Cain KL, et al. Environmental and demographic correlates of bicycling. Prev. Med. (Baltim). [Internet]. Elsevier B.V.; 2013 Jun 19 [cited 2013 Jul 1]; Available from: http://www.ncbi.nlm.nih.gov/pubmed/23791865

18. Garrard J, Rose G, Lo SK. Promoting transportation cycling for women: the role of bicycle infrastructure. Prev. Med. (Baltim). [Internet]. 2008 Jan [cited 2013 Oct 21];46(1):55-9. Available from: http://www.ncbi.nlm.nih.gov/pubmed/17698185

19. Brasil. Instituto Brasileiro de Geografia e Estatística. Sinopse dos Resultados do Censo 2010: distribuição da população por sexo, segundo os grupos de idade - Rio Claro-SP [Internet]. 2010. Available from: http://www.censo2010.ibge.gov.br/painel/?nivel=mn

20. Heinen E. Bicycle commuting. IOS Press. Amsterdam; 2011. p. 262.

21. Garrard J, Rose G, Lo SK. Promoting transportation cycling for women: the role of bicycle infrastructure. Prev. Med. (Baltim). [Internet]. 2008 Jan [cited 2013 Sep 25];46(1):55-9. Available from: http://www.ncbi.nlm.nih.gov/pubmed/17698185

22. Rissel C, Munro C, Baumann A. Assessing Cycling Participation in Australia. Sports [Internet]. 2013 Jan 2 [cited 2013 Sep 23];1(1):1-9. Available from: http://www.mdpi. com/2075-4663/1/1/1/

23. Rissel C, Merom D, Bauman A, Garrard J, Wen LM, New C. Current cycling, bicycle path use, and willingness to cycle more-findings from a community survey of cycling in southwest sydney, australia. J. Phys. Act. Health [Internet]. 2010 Mar;7(2):267-72. Available from: http:// www.ncbi.nlm.nih.gov/pubmed/20484766

24. Geus B De. Cycling to work Psychosocial and environmental factors associated Bas de Geus. Vrije Universiteit Brussel; 2007. p. 210.

25. Zacharias J. Non-motorized transportation in four Shanghai districts. Int. Plan. Stud. 2005;10(3):323-40. 
26. Lohmann G, Rolle D. I'd ride a bike but...!", changes in transport use from the background of the ipsative theory of behaviour. Umweltpsychologie. 2005;9(1):46-61.

27. Owen N, De Bourdeaudhuij I, Sugiyama T, Leslie E, Cerin E, Van Dyck D, et al. Bicycle Use for Transport in an Australian and a Belgian City: Associations with Built-Environment Attributes. J. Urban Heal. New York Acad. Med. [Internet]. 2010;87(2):189-98. Available from: <Go to ISI >://000276062100003

28. Engbers LH, Hendriksen IJ. Characteristics of a population of commuter cyclists in the Netherlands: perceived barriers and facilitators in the personal, social and physical environment. Int. J. Behav. Nutr. Phys. Act. [Internet]. BioMed Central Ltd; 2010 Jan [cited 2012 Jan 19];7(1):89. Available from: http://www.pubmedcentral.nih.gov/articlerender. fcgi?artid=3012015\&tool=pmcentre $\&$ rendertype $=$ abstract

29. Parkin J, Wardman M, Page M. Estimation of the determinants of bicycle mode share for the journey to work using census data. Transportation (Amst). [Internet]. 2008 Aug 3 [cited 2012 Jul 13];35(1):93-109. Available from: http://www.springerlink.com/index/10.1007/s11116007-9137-5

30. Winters M, Davidson G, Kao D, Teschke K. Motivators and deterrents of bicycling: comparing influences on decisions to ride. Transportation (Amst). [Internet]. 2010 Jun 13 [cited 2013 Sep 23];38(1):153-68. Available from: http://link.springer.com/10.1007/s11116-010-9284-y

31. Becerra JM, Reis RS, Frank LD, Ramirez-marrero FA, Welle B, Cordero EA, et al. Transport and health: a look at three Latin American cities. Cad. Saude Publica. 2013;29(4):654-66.

32. Pucher J, Komanoff C, Schimek P. Bicycling renaissance in North America ? Recent trends and alternative policies to promote bicycling. Transp. Res. Part A Policy Pract. [Internet]. 1999;33(7-8):625-54. Available from: http://www.sciencedirect.com/science/article/pii/ S0965856499000105

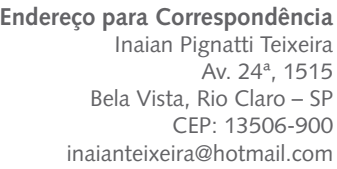

